Tainá J. Guimarães ${ }^{1}$

(O) https://orcid. org/0000-0001-7217-8033

Alessandra Perez

Ohtps://orid.org/0000-0003-4706-4952

Karin L. L. Dunker

OChtps://orcid.org/0000-0002-8686-7611

\section{Impacto de práticas parentais de peso e dieta na imagem corporal de adolescentes do sexo feminino}

\author{
Impact of weight and diet parenting practices on \\ body image of female adolescents
}

DOI: $10.1590 / 0047-2085000000262$

\section{RESUMO}

Objetivo: Avaliar as práticas parentais de peso e alimentação e sua relação com a insatisfação da imagem corporal em adolescentes. Métodos: As análises referem-se aos dados de base de um ensaio clínico randomizado previamente realizado com 270 adolescentes do sexo feminino de escolas públicas de São Paulo que participaram do Programa New Moves Brasil durante os anos de 2014 e 2015. Dados antropométricos, nível econômico, insatisfação com a imagem corporal, autoestima e práticas parentais sobre a ótica das adolescentes quanto à alimentação e corpo foram coletados. Um modelo de regressão logística múltipla foi conduzido para avaliar a influência das variáveis sobre a insatisfação com a imagem corporal das adolescentes. Resultados: Adolescentes que estavam acima do peso tinham média/baixa autoestima, sofriam provocações relativas ao peso e faziam menos refeições em família tiveram associação com a insatisfação com a imagem corporal. Na análise de regressão, somente o comentário sobre o peso da filha foi considerado como um fator de risco, e esse deixa de existir quando há o convívio de ambos os pais com as filhas. Em relação à influência do pai, no modelo de convivência familiar, ele pode ser tanto um modelo positivo, na influência de escolhas saudáveis, quanto negativo, ao fazer provocações sobre peso e o incentivar fazer dietas. Conclusões: Práticas parentais relacionadas ao peso e alimentação são consideradas de risco para o desenvolvimento de uma insatisfação com a imagem corporal e consequente predisposição a problemas relacionados ao peso.

\section{PALAVRAS-CHAVE}

Prática parental, obesidade, transtornos alimentares, adolescentes, imagem corporal.

\section{ABSTRACT}

Objective: To evaluate parenting practices of weight and diet and their relationship with body image dissatisfaction in adolescents. Methods: Data analysis refers to baseline data of a randomized clinical trial previously conducted with 270 female adolescents from public schools in São Paulo, who participated in the New Moves Brazil Program during 2014 and 2015. Anthropometric data, economic level, body dissatisfaction with body image, self-esteem, and parental practices of weight and diet from adolescents' perspective were collected. A multiple logistic regression model was conducted to evaluate the influence of the variables on the dissatisfaction with body image of adolescents. Results: Adolescents who were overweight, had medium/low self-esteem, suffered from weight teasing, and ate less family meals were associated with body image dissatisfaction. In the regression analysis, only comments about daughter's weight were considered as a risk factor that ceases to exist when both parents live with their daughters. Regarding influence of father, in a family coexistence model, he can be both a positive model, in the influence of healthy choices, and a negative one in teasing about weight and encouraging dieting. Conclusions: Parental practices related to weight and diet are considered as risk factor for the development of dissatisfaction with body image, and can consequently predispose to weight-related problems.

\section{KEYWORDS}

Parenting practice, obesity, eating disorders, adolescents, body image. 


\section{INTRODUÇÃO}

O sobrepeso e a obesidade pediátrica são considerados um problema mundial de saúde pública. A sua prevalência tem crescido consideravelmente em países em desenvolvimento, onde aproximadamente 13\% dos indivíduos com menos de 18 anos estão com sobrepeso ou obesidade'.

O excesso de peso é considerado um fator de risco para a obesidade na idade adulta, assim como determinante em curto e longo prazo no surgimento de doenças cardiovasculares, diabetes e alguns tipos de câncer. Além das consequências físicas, crianças e adolescentes acima do peso apresentam sofrimento emocional e baixa autoestima, já que encontram preconceito na escola $a^{2}$. Os prejuízos dessa condição podem ir além, por conta do desenvolvimento de uma insatisfação com a própria imagem corporal, que pode predispor à adoção de comportamentos inadequados de controle de peso (por exemplo, pular refeições, jejuar, tomar medicação para emagrecer, fumar cigarros, induzir vômitos, utilizar laxantes e diuréticos) e ao desenvolvimento de um transtorno alimentar ${ }^{3}$.

A forte evidência sobre a necessidade de encontrar estratégias efetivas de tratamento e realizar a prevenção da obesidade e dos transtornos alimentares tem levado os pesquisadores a não mais tratar esses problemas de forma separada, e sim conjuntamente, como problemas relacionados ao peso; ou seja, problemas que têm em comum fatores individuais e socioambientais que podem determinar o uso de métodos de controle de peso inadequados, insatisfação corporal, comer transtornado, compulsões alimentares e prática inadequada de atividade física.

Ações de prevenção voltadas a problemas relacionados ao peso têm sido estudadas ${ }^{5,6}$. O programa "New Moves" é considerado uma intervenção pioneira de prevenção integrada, com foco na mudança de comportamentos individuais modificáveis, como melhora do padrão de alimentação, redução do uso de métodos não saudáveis para controle de peso, melhora da imagem corporal, aumento da prática de atividade física e redução do sedentarismo, e não na perda de peso a curto prazo. Foi desenvolvido por pesquisadores da Universidade de Minnesota, nos Estados Unidos, e sua versão Brasileira - o New Moves Brasil (NMB) - foi adaptada e testada em adolescentes do sexo feminino de 12 a 14 anos de 10 de escolas públicas da cidade de São Paulo.

Seu delineamento e resultados já foram publicados anteriormente ${ }^{7}$, assim como o conteúdo do programa ${ }^{8}$. Além dos componentes individuais trabalhados no NMB, em módulos de suporte social, nutrição e atividade física, observou-se na experiência com o programa ${ }^{8}$ a importância de incluir a família nesse tipo de intervenção, uma vez que ela é considerada um importante fator socioambiental e de influência na promoção de hábitos alimentares saudáveis e na redução decomportamentos alimentares inadequados ${ }^{9}$.
Práticas parentais e seus efeitos nos comportamentos dos filhos têm sido discutidos pela literatura. A menor frequência de refeições em família ${ }^{10}$, o comportamento dos pais durante as refeições, como ser modelo de consumo de doces, preparar refeições especiais ${ }^{11}$, pressionar para comer menos gordura e insistir para comer alimentos saudáveis ${ }^{12}$ tem sido associado com uma maior predisposição ao ganho de peso. Estudos sobre outras práticas parentais, como a preocupação excessiva com o próprio peso e o dos filhos, a restrição alimentar própria e imposta aos filhos e a pressão para comer com uso de recompensa, apontam essas práticas como gatilhos determinantes de uma relação inadequada com a comida e corpo ao longo da vida de crianças e adolescentes ${ }^{13,14}$. O estudo longitudinal de Bauer ${ }^{15}$ reforça a importância dos pais em incentivar uma alimentação saudável e a prática de atividade física, mas desencoraja a pressão para fazer dieta e perder peso, que pode predispor a comportamentos de risco para desenvolver problemas relacionados ao peso.

Portanto, intervenções focadas na prevenção desses problemas devem incluir a participação dos pais, mas também o estudo anterior de práticas parentais que possam ser prejudiciais, para que ações educativas possam ser direcionadas a esse grupo e, dessa forma, influenciar de forma positiva a relação com comida e corpo de crianças e adolescentes. Em vista dos pontos apresentados, a hipótese de pesquisa do presente estudo, a ser testada a partir dos dados coletados pelo NMB, é que a insatisfação da imagem corporal em adolescentes do sexo feminino pode ser predita em modelos de regressões pelo estado nutricional (e.g., sobrepeso), nível econômico, autoestima e práticas parentais relacionadas à preocupação com o peso e à restrição alimentar.

\section{MÉTODOS}

Este estudo é baseado em uma análise secundária dos dados de base de um ensaio clínico randomizado previamente realizado com adolescentes do sexo feminino que participaram do NMB. Os termos de consentimento e assentimento foram assinados pelas adolescentes e seus pais/responsáveis antes da implementação do projeto que foi aprovado pelo Comitê de Ética da Universidade Federal de São Paulo (CAAE:06460112.6.0000.5505). Este estudo foi registrado no Brazilian Registry of Clinical Trials (registration number: RBR-6ddpb3).

O índice de massa corporal (IMC) foi calculado pela fórmula: peso em quilogramas dividido pela altura em metros ao quadrado. O peso foi medido por pessoal treinado do estudo usando uma balança digital, enquanto a estatura foi medida com um estadiômetro portátil. Com base nos percentis de IMC (IMC-P) padronizados por idade e sexo, os participantes foram classificados com baixo peso ou em risco 
(IMC-P < = 15), peso normal $(15<\mathrm{IMC}-\mathrm{P}<85)$, excesso de peso $(85<=\mid M C-P<97)$ ou obeso $(I M C-P>=97)^{16}$.

A "Classificação Econômica Brasileira"17 foi utilizada para classificar os estudantes de acordo com os estratos socioeconômicos. A soma desses escores determina a classe econômica da família, variando de A1 a E. Para fins de análise, geramos uma classificação dicotômica, a categoria média-alta, compreendendo as classes econômicas A2, B1 e B2, enquanto todas as outras foram classificadas como parte da categoria médio-baixa.

A insatisfação com a imagem corporal foi avaliada por meio de entrevista individual com o Questionário de Imagem Corporal (BSQ) ${ }^{18} \mathrm{e}$ a autoestima com a Escala de Autoestima de Rosenberg, ambas as escalas com versões adaptadas para adolescentes, tendo sido previamente validadas para o português ${ }^{19}$. Foram consideradas com algum grau de insatisfação (leve, moderado ou severo) meninas com ponto de corte maior que 80 no BSQ. A autoestima foi definida de acordo com uma pontuação no escore menor que 20 como média/baixa.

As participantes preencheram um questionário em que autorreferiam se concordam ou discordam quanto a práticas positivas dos pais - "Faz escolhas alimentares saudáveis", "Incentiva-me a ter uma dieta saudável" - e práticas negativas - "Faz dieta para perder peso", "Incentiva-me a fazer dietas para perder peso", "Comenta sobre o meu peso", "Comenta sobre o peso das outras pessoas". Referiram também se no último ano alguém de sua família fez provocações por causa do seu peso e aparência física, e quantas vezes na semana fez pelo menos uma refeição principal (café da manhã, almoço ou jantar) com a família. As perguntas fazem parte do questionário de avaliação do programa New Movess, que foram criadas a partir de um modelo de correlação entre fatores socioambientais e práticas de controle de peso inadequadas ${ }^{20}$. Foram traduzidas, adaptadas e validadas por Leme e Philippi ${ }^{21}$, em uma população de adolescentes do sexo feminino.

\section{ANÁLISE ESTATÍSTICA}

A distribuição das variáveis contínuas foi avaliada pelo teste Kolmogorov-Smirnov. Variáveis foram apresentadas como frequência absoluta e relativa, média e desvio-padrão ou mediana e intervalo interquartil, conforme apropriado. Testes de qui-quadrado, $t$ de Student e Mann-Whitney foram aplicados para analisar as diferenças entre os grupos, conforme apropriado. Um modelo de regressão logística múltipla foi conduzido para avaliar a influência das variáveis sobre o distúrbio da imagem corporal das adolescentes. Variáveis contínuas com distribuição assimétrica foram transformadas em logaritmo para a inclusão nos modelos. As análises foram realizadas por meio do programa SPSS, versão 23, e o p $<0,05$ foi estabelecido como limite para significância estatística.

\section{RESULTADOS}

Das 270 adolescentes alocadas no estudo randomizado, 11 $(4,1 \%)$ relataram não conviver com nenhum dos pais, portanto foram excluídas da presente análise. Do total de adolescentes incluídas ( $n=259), 250$ referiram conviver com a mãe, 136 com o pai e 127 com ambos.

Na tabela 1, observa-se que a maioria das adolescentes pertence à classe média/baixa, não apresenta grau de insatisfação com a imagem corporal $(63,6 \%, n=164)$ e tem média/baixa autoestima $(71,8 \%, n=186)$. As participantes com algum grau de insatisfação com a imagem corporal apresentavam maior: IMC, frequência de comprometimento da autoestima, relato de a família fazer provocações por causa do peso e aparência física, além de realizarem menos refeições na presença de familiares ao longo da semana quando comparadas àquelas sem grau de insatisfação.

Na percepção das meninas, a mãe tem mais preocupação com o peso e a alimentação dela mesma e com a filha do que o pai (Tabela 2). Por exemplo, 41,6\% das mães fazem dieta para perder peso e 52,4\% comentam sobre o peso da filha contra $19,8 \%$ e $31,6 \%$ dos pais, respectivamente.

Quando avaliadas em relação à presença ou não de insatisfação com a imagem corporal e sobre práticas dos pais em relação à alimentação e ao corpo (Tabela 2), observa-se que adolescentes com insatisfação reportaram com maior frequência o incentivo para manter uma dieta saudável, realizar dietas para perder peso, sofrer comentários sobre o seu peso e perceber comentários sobre o peso de outras pessoas, por parte da mãe. Em relação ao pai, a influência é menos frequente, somente na percepção sobre praticar escolhas alimentares saudáveis e incentivo à realização de dietas para perder peso.

Na tabela 3, observa-se que, em todos os modelos de regressão logística aplicados sobre a percepção sobre a mãe (modelo 1), o pai (modelo 2) ou ambos (modelo 3), o IMC elevado e o comprometimento da autoestima estiveram independentemente associados à insatisfação com a imagem corporal.

No modelo 2, observa-se que fazer provocações sobre o peso e incentivar a filha a fazer dietas na percepção das meninas sobre o pai é considerado fator de risco significativo para a insatisfação corporal, que se mantém no modelo 3 da percepção de ambos. Também foi observado que as escoIhas alimentares saudáveis do pai têm influência como fator protetor. Em relação à percepção das meninas sobre a mãe (modelo 1), observa-se que comentar sobre o peso da filha é um fator de risco, mas que não se mantém quando há o convívio com pai e mãe. 
Tabela 1. Características da população de acordo com a insatisfação com a imagem corporal

\begin{tabular}{|c|c|c|c|c|c|}
\hline & $\begin{array}{c}\text { Total } \\
(\mathrm{n}=259)\end{array}$ & $\begin{array}{l}\text { Com insatisfação } \\
\qquad(\mathrm{n}=95)\end{array}$ & $\begin{array}{l}\text { Sem insatisfação } \\
\quad(n=164)\end{array}$ & $\mathrm{X}^{2}, \mathrm{Z}$ ou U & $\mathbf{P}$ \\
\hline Idade (anos) & $13,4 \pm 0,6$ & $13,5 \pm 0,7$ & $13,4 \pm 0,6$ & $-1,065$ & 0,264 \\
\hline Peso (kg) & $51(44-60)$ & $58(52-66)$ & $47(42-54)$ & $-7,353$ & 0,000 \\
\hline Altura (m) & $1,58(1,53-1,63)$ & $1,56(1,52-1,61)$ & $1,59(1,55-1,64)$ & $-3,193$ & 0,001 \\
\hline IMC $\left(\mathrm{kg} / \mathrm{m}^{2}\right)$ & $20,3(18,3-23,6)$ & $22,7(20,2-25,5)$ & $19,2(17,5-22,3)$ & $-6,445$ & 0,000 \\
\hline Sobrepeso/obesidade [n(\%)] & $88(34,0)$ & $47(49,5)$ & $41(25,0)$ & 16,062 & 0,000 \\
\hline Classe social média/baixa [n (\%)] & $157(60,6)$ & $57(60,0)$ & $100(61,0)$ & 0,024 & 0,877 \\
\hline Questionário de imagem corporal (pontos) & $65(47-102)$ & $112(95-126)$ & $49(42-61)$ & $-13,410$ & 0,000 \\
\hline Escala de Rosenberg (pontos) & $18(15-21)$ & $16(14-19)$ & $19(17-22)$ & $-5,697$ & 0,000 \\
\hline Média/baixa autoestima [n (\%)] & $186(71,8)$ & $81(85,3)$ & $105(64,0)$ & 13,406 & 0,000 \\
\hline Sofreu brincadeiras sobre o peso [n (\%)] & $77(29,7)$ & $43(45,3)$ & $34(20,7)$ & 17,328 & 0,000 \\
\hline Refeições feitas em família (n/semana) & $4(2-7)$ & $3(1-7)$ & $5(2-7)$ & $-2,122$ & 0,034 \\
\hline Convive com a mãe [n (\%)] & $250(96,5)$ & $90(94,7)$ & $160(97,6)$ & 1,430 & 0,232 \\
\hline Convive com o pai [n (\%)] & $136(52,5)$ & $49(51,6)$ & $87(53,0)$ & 0,052 & 0,819 \\
\hline Convive com a mãe e o pai [n (\%)] & $127(49,0)$ & $44(46,3)$ & $83(50,6)$ & 0,444 & 0,505 \\
\hline
\end{tabular}

Tabela 2. Percepção das adolescentes sobre as práticas dos pais em relação à alimentação e ao corpo

\begin{tabular}{|c|c|c|c|c|c|c|c|c|c|c|}
\hline & \multicolumn{5}{|c|}{$\begin{array}{l}\text { Percepção sobre a mãe } \\
\qquad(\mathrm{n}=\mathbf{2 5 0})\end{array}$} & \multicolumn{5}{|c|}{$\begin{array}{l}\text { Percepção sobre o pai } \\
\quad(n=136)\end{array}$} \\
\hline & Total & $\begin{array}{c}\text { Com } \\
\text { insatisfação }\end{array}$ & $\begin{array}{c}\text { Sem } \\
\text { insatisfação }\end{array}$ & $\mathbf{X}^{2}$ & $\mathbf{P}$ & Total & $\begin{array}{c}\text { Com } \\
\text { insatisfação }\end{array}$ & $\begin{array}{c}\text { Sem } \\
\text { insatisfação }\end{array}$ & $\mathbf{X}^{2}$ & p \\
\hline $\begin{array}{l}\text { Faz escolhas alimentares } \\
\text { saudáveis }\end{array}$ & $202(80,8)$ & $74(82,2)$ & $128(80,0)$ & 0,183 & 0,669 & $77(56,6)$ & $21(42,9)$ & $56(64,4)$ & 5,905 & 0,015 \\
\hline $\begin{array}{l}\text { Incentiva-me a ter uma dieta } \\
\text { saudável }\end{array}$ & $209(83,6)$ & $83(92,2)$ & $126(78,8)$ & 7,625 & 0,006 & $92(67,6)$ & $35(71,4)$ & $57(65,5)$ & 0,500 & 0,479 \\
\hline Faz dieta para perder peso & $104(41,6)$ & $41(45,6)$ & $63(39,4)$ & 0,906 & 0,341 & $27(19,8)$ & $12(24,5)$ & $15(17,2)$ & 1,035 & 0,309 \\
\hline $\begin{array}{l}\text { Incentiva-me a fazer dietas para } \\
\text { perder peso }\end{array}$ & $88(35,2)$ & $51(56,7)$ & $37(23,1)$ & 28,410 & 0,000 & $33(24,2)$ & $23(46,9)$ & $10(11,5)$ & 21,429 & 0,000 \\
\hline Comenta sobre o meu peso & $131(52,4)$ & $65(72,2)$ & $66(41,3)$ & 22,153 & 0,000 & $43(31,6)$ & $18(36,7)$ & $25(28,7)$ & 0,928 & 0,335 \\
\hline $\begin{array}{l}\text { Comenta sobre o peso das } \\
\text { outras pessoas }\end{array}$ & $30(12,0)$ & $28(31,1)$ & $32(20,0)$ & 3,899 & 0,048 & $21(15,4)$ & $9(18,4)$ & $12(13,8)$ & 0,502 & 0,478 \\
\hline
\end{tabular}

\section{DISCUSSÃO}

Observou-se na caracterização da amostra que estar acima do peso, ter média/baixa autoestima, sofrer provocações quanto ao peso e fazer menos refeições em família foi associado com insatisfação corporal. Apesar de as práticas da mãe em relação a peso e dieta apresentarem uma maior influência na insatisfação corporal do que as do pai, na análise de regressão somente o comentário sobre o peso da filha foi considerado como um fator de risco, e este deixa de existir quando há o convívio de ambos os pais com as filhas. Em relação à influência do pai, no modelo de convivência familiar, ele pode ser tanto um modelo positivo, na influência de escolhas saudáveis, quanto negativo, ao fazer brincadeiras sobre peso e incentivar fazer dietas.

Estudos indicam que a família fazer provocações sobre o peso é um dos fatores mais importantes na gênese de problemas relacionados ao peso $0^{3,21,22}$. E além de terem maior insatisfação, também têm risco de adotar métodos de controle de peso extremos e não saudáveis, e de apresentar episódios de compulsão alimentar ${ }^{3,23}$.

Fazer refeições em família também tem sido associado com menor risco de métodos compensatórios como purgação, compulsão alimentar e hábito de fazer dieta. Também ajuda a melhorar a qualidade da relação de pais e filhos, em particular na comunicação e coesão, o que ajuda na redução do risco em se envolver em comportamentos desordenados ${ }^{9,24}$.

Evidências da literatura apontam a mãe como maior modelo de prática de dietas, de insatisfação com o próprio peso/corpo, que pode determinar um maior desejo das fiIhas pela busca da magreza ${ }^{25,26}$. Cooley et al. ${ }^{27}$ e Bauer et al. ${ }^{28}$ apontam que dois mecanismos poderiam explicar o efeito dessas práticas: um em que a mãe encorajaria de forma 
Tabela 3. Modelo de regressão logística sobre fatores de risco para a insatisfação com a imagem corporal, na percepção sobre a mãe, o pai ou ambos

\begin{tabular}{|c|c|c|c|c|c|c|c|}
\hline \multirow{2}{*}{ Modelo 1. Percepção sobre a mãe $(n=250)$} & \multirow{2}{*}{ B } & \multirow{2}{*}{ S.E } & \multirow{2}{*}{ Wald } & \multirow{2}{*}{ OR } & \multicolumn{2}{|c|}{ IC (95\%) } & \multirow{2}{*}{$\mathbf{p}$} \\
\hline & & & & & Inferior & Superior & \\
\hline IMC (kg/m²) & 0,147 & 0,045 & 10,713 & 1,158 & 1,061 & 1,265 & 0,001 \\
\hline Autoestima comprometida & 1,596 & 0,416 & 14,722 & 4,933 & 2,183 & 11,147 & 0,000 \\
\hline Sofreu brincadeiras sobre o peso [n (\%)] & $-0,584$ & 0,343 & 2,898 & 0,558 & 0,285 & 1,092 & 0,089 \\
\hline Refeições feitas em família (n\%semana) & $-0,058$ & 0,059 & 0,977 & 0,943 & 0,840 & 1,059 & 0,323 \\
\hline Incentiva-me a ter uma dieta saudável & 0,587 & 0,502 & 1,368 & 1,799 & 0,672 & 4,816 & 0,242 \\
\hline Incentiva-me a fazer dietas para perder peso & 0,702 & 0,362 & 3,768 & 2,018 & 0,993 & 4,102 & 0,052 \\
\hline Comenta sobre o meu peso & 0,756 & 0,332 & 5,202 & 2,130 & 1,112 & 4,079 & 0,023 \\
\hline Comenta sobre o peso das outras pessoas & 0,465 & 0,358 & 1,688 & 1,592 & 0,789 & 3,211 & 0,194 \\
\hline \multirow{2}{*}{ Modelo 2. Percepção sobre o pai $(n=136)$} & \multirow{2}{*}{ B } & \multirow{2}{*}{ S.E } & \multirow{2}{*}{ Wald } & \multirow{2}{*}{$\mathbf{O R}$} & \multicolumn{2}{|c|}{ IC (95\%) } & \multirow{2}{*}{$\mathbf{p}$} \\
\hline & & & & & Inferior & Superior & \\
\hline $\mathrm{IMC}\left(\mathrm{kg} / \mathrm{m}^{2}\right)^{*}$ & 0,132 & 0,060 & 4,871 & 1,141 & 1,015 & 1,284 & 0,027 \\
\hline Autoestima comprometida & $-1,866$ & 0,606 & 9,495 & 0,155 & 0,047 & 0,507 & 0,002 \\
\hline Sofreu brincadeiras sobre o peso [n (\%)] & 1,582 & 0,514 & 9,476 & 4,867 & 1,777 & 13,330 & 0,002 \\
\hline Refeições feitas em família (n\%semana) & $-0,019$ & 0,089 & 0,047 & 0,981 & 0,823 & 1,169 & 0,828 \\
\hline Faz escolhas alimentares saudáveis & $-1,358$ & 0,495 & 7,527 & 0,257 & 0,098 & 0,679 & 0,006 \\
\hline Incentiva-me a fazer dietas para perder peso & 1,849 & 0,585 & 9,997 & 6,352 & 2,019 & 19,983 & 0,002 \\
\hline \multirow{2}{*}{ Modelo 3. Percepção sobre ambos $(n=127)$} & \multirow{2}{*}{ B } & \multirow{2}{*}{ S.E } & \multirow{2}{*}{ Wald } & \multirow{2}{*}{ OR } & \multicolumn{2}{|c|}{ IC (95\%) } & \multirow{2}{*}{$\mathbf{p}$} \\
\hline & & & & & Inferior & Superior & \\
\hline IMC $\left(\mathrm{kg} / \mathrm{m}^{2}\right)^{*}$ & 0,148 & 0,070 & 4,525 & 1,160 & 1,012 & 1,330 & 0,033 \\
\hline Autoestima comprometida & $-1,890$ & 0,659 & 8,228 & 0,151 & 0,042 & 0,550 & 0,004 \\
\hline Sofreu brincadeiras sobre o peso [n (\%)] & 1,151 & 0,558 & 4,257 & 3,160 & 1,059 & 9,428 & 0,039 \\
\hline Refeições feitas em família (n\%semana) & $-0,003$ & 0,099 & 0,001 & 0,997 & 0,821 & 1,210 & 0,973 \\
\hline Faz escolhas alimentares saudáveis (pai) & $-1,625$ & 0,571 & 8,112 & 0,197 & 0,064 & 0,602 & 0,004 \\
\hline Incentiva-me a fazer dietas para perder peso (pai) & 1,826 & 0,735 & 6,172 & 6,208 & 1,470 & 26,218 & 0,013 \\
\hline Incentiva-me a ter uma dieta saudável (mãe) & 1,281 & 0,940 & 1,856 & 3,601 & 0,570 & 22,745 & 0,173 \\
\hline Incentiva-me a fazer dietas para perder peso (mãe) & $-0,548$ & 0,679 & 0,652 & 0,578 & 0,153 & 2,186 & 0,419 \\
\hline Comenta sobre o meu peso (mãe) & 0,629 & 0,561 & 1,257 & 1,875 & 0,625 & 5,628 & 0,262 \\
\hline Comenta sobre o peso das outras pessoas (mãe) & 0,553 & 0,595 & 0,864 & 1,739 & 0,541 & 5,587 & 0,353 \\
\hline
\end{tabular}

direta a filha a mudar o peso ou forma e o outro em que a mãe de forma indireta encoraja por meio de seus comentários sobre seu próprio peso e seu comportamento de fazer dieta. Apesar dos autores apontarem esses mecanismos em conjunto como determinantes de maior insatisfação corporal nas filhas, estudos indicam que o incentivo a fazer dieta tem mais efeito no desejo por emagrecer nas filhas do que os comentários sobre o próprio peso da mãe ${ }^{25,26}$.

Ainda não é totalmente clara a forma como os pais influenciam as filhas ${ }^{3,29}$. Alguns estudos verificaram que práticas dos pais de restrição alimentar e controle estiveram associados com maior peso das crianças ${ }^{30}$. Um estudo sobre fatores de risco para preocupação com peso e busca pela magreza indica que a reação que o pai tem de insatisfação com seu próprio peso pode por si só ser considerada um risco. Essa insatisfação pode torná-lo mais sensível e crítico ao peso da filha e esposa, desaprovando o excesso de peso de forma direta ou indireta ${ }^{31}$.
Se por um lado uma das limitações do estudo é a avaliação das práticas parentais sob a ótica das filhas, por outro, estudos empíricos indicam que as percepções sobre os comportamentos dos pais pelos filhos estão mais fortemente associadas com o comportamento da criança. Ou seja, crianças tendem a copiar o comportamento dos pais e, dessa forma, o que registram e respondem podem ser respostas mais realistas do que a própria autopercepção dos pais ${ }^{22}$. Outras limitações incluem o tamanho da amostra, o fato de ter sido aplicado somente em meninas e em uma faixa etária restrita e os resultados não indicarem causa e efeito.

Os resultados indicam que as práticas parentais pela percepção das filhas podem ter influências diferentes na insatisfação corporal e que, portanto, tanto a mãe como o pai devem ser incluídos em ações na prevenção de problemas relacionados ao peso em crianças e adolescentes. Essas intervenções devem focar no incentivo às refeições em família, atentando para a importância do modelo dos pais à mesa, 
evitando o incentivo à restrição alimentar e comentários sobre peso e forma. Os pais devem estimular um ambiente de tranquilidade, comunicação e aceitação, para que, dessa forma, se construa uma autoestima e autoimagem positiva ${ }^{32}$.

\section{CONCLUSÕES}

Os resultados indicam que os comportamentos disfuncionais dos pais quanto a alimentação, e peso podem gerar uma insatisfação corporal nas filhas. Justifica-se a necessidade de investigações futuras sobre o efeito da comunicação positiva relacionada a peso, alimentação e imagem corporal na redução ou ausência de comportamentos de risco para os problemas relacionados ao peso.

\section{CONTRIBUIÇÕES INDIVIDUAIS}

Tainá J. Guimarães - (1) Contribuiu significativamente na análise e interpretação dos dados; (2) contribuiu substancialmente na elaboração do artigo; (3) aprovou a versão final a ser publicada.

Alessandra Perez - (1) Contribuiu significativamente na análise e interpretação dos dados; (2) contribuiu substancialmente na elaboração do artigo; (3) aprovou a versão final a ser publicada.

Karin L. L. Dunker - (1) Contribuiu significativamente na concepção e desenho dos estudos ou na análise e interpretação dos dados; (2) contribuiu substancialmente na elaboração do artigo ou revisando criticamente o seu conteúdo intelectual; (3) aprovou a versão final a ser publicada.

\section{CONFLITO DE INTERESSES}

Declaramos que não há conflito de interesses entre os autores deste artigo.

\section{AGRADECIMENTOS}

Fundação de Amparo à Pesquisa do Estado de São Paulo (Fapesp) (processo número: 2012/16952-8) e Conselho Nacional de Desenvolvimento Científico e Tecnológico (CNPq) (processo número: 483871/2013-3).

\section{REFERÊNCIAS}

1. Ng M, Fleming T, Robinson M, Thomson B, Graetz N, Margono C, et al. Global, regional, and national prevalence of overweight and obesity in children and adults during 1980-2013: a systematic analysis for the Global Burden of Disease Study. Lancet. 2014;384(9945):766-81.
2. Reilly JJ, Kelly J. Long-term impact of overweight and obesity in childhood and adolescence on morbidity and premature mortality in adulthood: systematic review. Int I Obes (Lond). 2011;35(7):891-8.

3. Neumark-Sztainer D, Bauer KW, Friend S, Hannan PJ, Story M, Berge JM. Family weight talk and dieting: How much do they matter for body dissatisfaction and disordered eating behaviors in adolescent girls? J Adolesc Health. 2010;47(3):270-6.

4. Sánchez-Carracedo D, Neumark-Sztainer D, López-Guimera G. Integrated prevention of obesity and eating disorders: barriers, developments and opportunities. Public Health Nutr. 2012;15(12):2295-309.

5. Neumark-Sztainer D, Friend SE, Flattum CF, Hannan PJ, Story MT, Bauer KW, et al. New moves-preventing weight-related problems in adolescent girls a group-randomized study. Prev Med. 2010;39(5):421-32.

6. Stice E, Rohde P, Shaw H, Gau JM. An experimental therapeutics test of whether adding dissonance-induction activities improves the effectiveness of a selective obesity and eating disorder prevention program. Int J Obes (Lond). 2018;42(3):462-8.

7. Dunker KLL, Claudino AM. Preventing weight-related problems among adolescent girls: A cluster randomized trial comparing the Brazilian 'New Moves' program versus observation. Obes Res Clin Pract. 2018;12(1):102-15.

8. Dunker KLL, Alvarenga MS, Claudino AM. Prevenção de transtornos alimentares e obesidade: relato de experiência da implementação do programa New Moves. Saúde Debate. 2018:42(116):331-42.

9. Neumark-Sztainer D, Eisenberg ME, Fulkerson JA, Story M, Larson NI. Family meals and disordered eating in adolescents: longitudinal findings from project EAT. Arch Pediatr Adolesc Med. 2008;162(1):17-22.

10. Tosatti AM, Ribeiro LW, Machado RHV, Maximimo P, Bozzini AB, Ramos CC, et al. Does family mealtime have a protective effect on obesity and good eating habits in young people? A 2000-2016 review. Rev Bras Saude Mater Infant. 2017;17(3):425-34.

11. Melo KM, Cruz ACP, Brito MFSF, Pinho L. Influence of parents' behavior during the meal and on overweight in childhood. Esc Anna Nery. 2017;21(4).

12. Petty MLB, Schimith-Escrivão MA, Kyong-Mee C, Jung WH, Hendy HM, Williams KE. Comparison of maternal feeding practices and child weight status in children from three countries. Int J Child Health Nutrition. 2014;3:67-77.

13. Loth K, Fulkerson JA, Neumark-Sztainer D. Food-related parenting practices and child and adolescent weight and weight-related behaviors. Clin Pract (Lond). 2014;11(2):207-20.

14. Lydecker JA, Riley KE, Grilo CM. Associations of parents' self, child, and other "fat talk" with child eating behaviors and weight. Int J Eat Disord. 2018;51(6):527-34.

15. Bauer KW, Laska MN, Fulkerson JA, Neumark-Sztainer D. Longitudinal and secular trends in parental encouragement for healthy eating, physical activity, and dieting throughout the adolescent years. J Adolesc Health. 2011;49(3):306-11.

16. World Health Organization (WHO). Growth reference data for 5-19 years. Geneve: WHO; 2007.

17. Empresas de Pesquisa $A B$ de. Critério de classificação econômica Brasil. Disponível em: http://www.abeporg/servicos/downloadaspx?id=11. Acesso em: 28 jul. 2016.

18. Conti MA, Cordás TA, Latorre MRDO. Estudo de validade e confiabilidade da versão brasileira do Body Shape Questionnaire (BSQ) para adolescentes. Rev Bras Saude Mater Infant. 2009;9(3):331-8.

19. Avanci JQ, Assis SG, Santos NC, Oliveira RVC. Adaptação tanscultural de escala de autoestima para adolescentes. Psicol Reflex Crit. 2007;20(3):397-405.

20. Neumark-Sztainer D, Wall MM, Story M, Perry CL. Correlates of unhealthy weight-control behaviors among adolescents: implications for prevention programs. Health Psychol. 2003;22(1):88-98,

21. Leme ACB, Philippi ST. Provocações e comportamentos para controle de peso em adolescentes do sexo feminino. Rev Paul Pediatr. 2013;31(4):431-6.

22. Keery H, Boutelle K, van den Berg P, Thompson JK. The impact of appearance-related teasing by family members. J Adolesc Health. 2005;37(2):120-7.

23. Menzel JE, Schaefer LM, Burke NL, Mayhew LL, Brannick MT, Thompson JK. Appearancerelated teasing, body dissatisfaction, and disordered eating: a meta-analysis. Body Image. 2010; $7(4): 261-70$.

24. Haines J, Gillman MW, Rifas-Shiman S, Field AE, Austin SB. Family dinner and disordered eating behaviors in a large cohort of adolescents. Eat Disord. 2010;18(1):10-24. 
25. Klein KM, Brown TA, Kennedy GA, Keel PK. Examination of parental dieting and comments as risk factors for increased drive for thinness in men and women at 20-year follow-up. Int J Eat Disord. 2017;50(5):490-7.

26. Hillard EE, Gondoli DM, Corning AF, Morrissey RA. In it together: Mother talk of weight concerns moderates negative outcomes of encouragement to lose weight on daughter body dissatisfaction and disordered eating. Body Image. 2016;16:21-7.

27. Cooley E, Toray T, Wang MC, Valdez NN. Maternal effects on daughters' eating pathology and body image. Eat Behav. 2008;9(1):52-61.

28. Bauer A, Schneider S, Waldorf M, Adolph D, Vocks S. Familial transmission of a body-related attentional bias - An eye-tracking study in a nonclinical sample of female adolescents and their mothers. PLoS One. 2017;12(11):e0188186.
29. Snoek HM, van Strien T, Janssens JM, Engels RC. Longitudinal relationships between fathers', mothers', and adolescents' restrained eating. Appetite. 2009;52(2):461-8.

30. Loth K, MacLehose R, Fulkerson J, Crow S, Neumark-Sztainer D. Food-related parenting practices and adolescent weight status: a population based study. Pediatrics. 2013;131(5):e1443-50

31. Agras WS, Bryson S, Hammer LD, Kraemer HC. Childhood risk factors for thin body preoccupation and social pressure to be thin. J Am Acad Child Adolesc Psychiatry. 2007;46(2):171-8.

32. Hart LM, Cornell C, Damiano SR, Paxton SJ. Parents and prevention: A systematic review of interventions involving parents that aim to prevent body dissatisfaction or eating disorders. Int J Eat Disord. 2015;48(2):157-69. 